\title{
Big fish, little fish
}

\author{
Dirk J. Varelmann, MD, and Michael N. D’Ambra, MD
}

\footnotetext{
From the Department of Anesthesiology, Perioperative and Pain Medicine, Brigham and Women's Hospital, Boston, Mass.

Disclosures: Authors have nothing to disclose with regard to commercial support.

Received for publication May 24, 2016; accepted for publication May 26, 2016; available ahead of print June 18, 2016.

Address for reprints: Dirk J. Varelmann, MD, Department of Anesthesiology, Perioperative and Pain Medicine, Brigham and Women's Hospital, 75 Francis St, CWN-L1, Boston, MA 02115 (E-mail: dvarelmann@ partners.org).

J Thorac Cardiovasc Surg 2016;152:890

$0022-5223 / \$ 36.00$

Copyright $(\underset{2}{ } 2016$ by The American Association for Thoracic Surgery

http://dx.doi.org/10.1016/j.jtcvs.2016.05.047
}

Mamoun and colleagues at the Cleveland Clinic Foundation conducted a highly sophisticated study ${ }^{1}$ of the effectiveness of intravenous (IV) acetaminophen in patients undergoing median sternotomy for cardiac surgical operations. The study was funded by a grant from Cadence Pharmaceutical.

Acetaminophen IV, given in regular doses starting at the end of cardiopulmonary bypass and extending into the intensive care unit period, improves pain scores but does not reduce the need for supplemental opioids via patient-controlled analgesia. The scientific findings are clear and supported by robust statistical analysis.

Although the reduction in pain by 1 point measured on a 11-point Numeric Rating Scale was statistically significant, it may not be clinically significant. Farrar and colleagues ${ }^{2}$ consider a reduction of 2 points or $30 \%$ on the Numeric Rating Scale as a clinically important difference. Other clinical benefits could not be established in this study: IV acetaminophen did not reduce the amount of opioids needed, nor did it decrease the duration of mechanical ventilation or length of stay in the intensive care unit/ hospital.

Clearly, IV acetaminophen will increase the drug expenses, although it is unknown by how much. The authors were asked this question by multiple reviewers, and they were not responsive. The authors have no declared conflict of interest, but they were unwilling to include a cost analysis or even to comment in the manuscript on the reasons why they think a cost analysis is not possible in the United States. However, they did state these reasons in their response to the reviewers. One reason was that every hospital has different pricing, which the hospital pharmacies negotiate and are unwilling to disclose. Another is the fact that during the study, Mallinckrodt PLC bought Cadence and quadrupled the price of the drug in the United States.

After reading this manuscript, surgeons and intensivists are left with the technical possibility of an improvement journal publication.

\section{References}

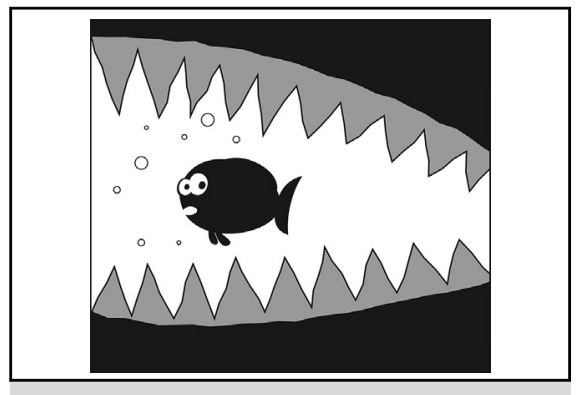

Big Pharma acquisitions increase cost of care.

Central Message

Intravenous acetaminophen for cardiac surgery delivers a clinically insignificant improvement in surgical pain at a significant increase in drug expense.

See Article page 881

in the comfort of the patient with no way to understand whether it will reduce or increase the cost of care. Certainly the quadrupling of the cost of this drug is quite disturbing, especially as the benefits for the patients may not be clinically significant or simply don't exist. It is time for the academic community (meaning institutional review boards) to take a stand and for high impact-factor journals like this one to start requiring that industry-sponsored device and drug trials include responsible and comprehensible cost analyses as part of the protocols as a prerequisite for institutional review board approval and

In countries like Canada and the United Kingdom, government dictates the price it will pay nationwide, and cost analysis become easier to do for drugs that have salutary effects on therapeutic and safety outcomes. Thus, physicians in those countries can make informed decisions about adding therapies like IV acetaminophen to the process of care. In the United States, Big Pharma makes such decision making nearly impossible.

1. Mamoun NF, Lin P, Zimmerman NM, Mascha EJ, Mick SL, Insler SR. Intravenous acetaminophen analgesia after cardiac surgery: a randomized, blinded controlled superiority trial. J Thorac Cardiovasc Surg. 2016;152:881-9.e1.

2. Farrar JT, Young JPJ, LaMoreaux L, Werth JL, Poole RM. Clinical importance of changes in chronic pain intensity measured on an 11-point numerical pain rating scale. Pain. 2001;94:149-58. 